\title{
Registro de diatomeas de los últimos 1400 años de la Laguna Pululos, Jujuy, Argentina
}

\author{
Marcelo R. Morales ${ }^{1, \otimes ;}$; Sabrina Bustos² \& Nora I. Maidana ${ }^{1}$
}

\begin{abstract}
${ }^{1} I B B E A, C O N I C E T$ - UBA. Laboratorio de Diatomeas Continentales, DBBE, FCEN, UBA, Ciudad Universitaria, Pab. 2, $4^{\circ}$ piso, Lab. 15 (C1428EHA) Ciudad Autónoma de Buenos Aires, Argentina. ${ }^{2}$ Laboratorio de Diatomeas Continentales, DBBE, FCEN, UBA, Ciudad Universitaria, Pab. 2, $4^{\circ}$ piso, Lab. 15. (C1428EHA) Ciudad Autónoma de Buenos Aires, Argentina.
\end{abstract}

\begin{abstract}
RESUMEN. En este trabajo se presenta información paleoambiental de alta resolución cronológica (multi-decadal) de los últimos 1400 años para el sector noroeste de la Puna seca de la provincia de Jujuy (Argentina). Esta información fue obtenida a partir del análisis de la composición de los ensambles de diatomeas recuperados de

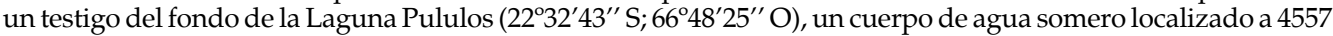
m.s.n.m. Se describen y discuten los resultados de este análisis y las tendencias paleoambientales observadas en relación a las características y cronologías de la anomalía climática medieval (ACM) y de la pequeña edad de hielo (PEH). Entre los años 800 y 1500 DC se habrían instalado marcadas condiciones de aridez, seguidas por lapsos más húmedos que se prolongaron hasta fines del siglo XIX. Se destacan dos períodos: entre 800 y 600 DC (la fase más húmeda de los últimos 1400 años) y entre 1366 y 1463 DC (especialmente árido). Si bien la Laguna Pululos habría atravesado etapas con características asimilables a las del ACM y a las de la PEH, diferiría con el hemisferio norte en cuanto a su cronología, presentando una duración mayor de las condiciones de aridez de la ACM respecto a otros sitios estudiados en los Andes.
\end{abstract}

[Palabras clave: paleoambiente, paleolimnología, resolución decadal, Puna seca, Andes Tropicales, holoceno tardío, anomalía climática medieval, pequeña edad de hielo, ficología, Complejo Vilama]

\begin{abstract}
Aвstract. Diatom record of the last 1400 years in Laguna Pululos Jujuy, Argentina. This paper presents high-resolution (multi-decadal) paleoenvironmental data of the last 1400 years in the northwestern sector of the dry Puna, Jujuy Province (Argentina). This information results from the analysis of the diatom assemblies recovered from sediment core samples collected at Laguna Pululos $\left(22^{\circ} 32^{\prime} 43^{\prime \prime}\right.$ S; $66^{\circ} 48^{\prime} 25^{\prime \prime}$ W), a shallow lake located at 4557 m.a.s.l. In this work we present and discuss the main results regarding the species composition of the diatom assemblages, and the observed paleoenvironmental trends related to the main characteristics and chronology of the medieval climatic anomaly (MCA) and little ice age (LIA). The results of the study indicate the onset of very arid conditions between $\sim 800$ and $1500 \mathrm{AD}$, and a sharp change to moister conditions from the latter period until the end of the XIX century. Noteworthy, two periods could be recognized, one between 600 and $800 \mathrm{AD}$ (the moistest phase of the last 1400 years), and between 1366 and $1463 \mathrm{AD}$ (a particularly arid phase). These periods recorded in Laguna Pululos apparently shared characteristics with the MCA and LIA, although, showing differences with chronologies found in the North Hemisphere, with an extended span of the arid conditions during the MCA in comparison with other paleoenvironmental records of the Andes.
\end{abstract}

[Keywords: paleoenvironment, paleolimnology, decadal resolution, dry Puna, Tropical Andes, late holocene, medieval climatic anomaly, little ice age, phycology, Vilama Complex]

\section{INTRODUCCIÓN}

En muchos de los registros paleoambientales que abarcan los últimos 2000 años a lo largo del planeta se han detectado dos cronozonas que representan cambios climáticos y/o ambientales significativos. La primera ocurrió entre los siglos VIII y XII, y la segunda comenzó alrededor del siglo XV y finalizó en las postrimerías del siglo XIX. Estas cronozonas son las denominadas anomalía climática medieval (ACM) (también conocida como calentamiento vikingo $\mathrm{u}$ óptimo medieval) y la pequeña edad de hielo (PEH). Ambos períodos están bien documentados, en particular en el hemisferio norte, aunque sus

\section{Editora asociada: María Dieguez}

$\triangle$ marcelomoralesarq@gmail.com características principales, tanto en lo que hace a su duración y cronología exacta como a su impacto ambiental concreto (e.g., temperatura, humedad, etc.), suelen diferir de manera notable entre las localidades estudiadas (Díaz et al. 2011).

En términos generales, la ACM fue descripta para el hemisferio norte como un período muy cálido, que instaló condiciones de aridez en la Europa mediterránea y favoreció, en términos climáticos, al sector septentrional de Europa y a la península escandinava (como lo documentan numerosas fuentes históricas [Lamb 1965]). Estas condiciones permitieron el desarrollo de cultivos como la vid en las

Recibido: 17 de marzo de 2015, Fin de arbitraje: 28 de mayo de 2015, Última versión revisada: 18 de junio, Aceptado: 25 de julio de 2015. 
islas británicas, habilitaron condiciones óptimas para las exploraciones vikingas de las costas occidentales del Mar del Norte, incluyendo el sector oriental de la Península de Labrador en Norteamérica, y generaron cosechas con resultados extraordinarios en la mayor parte de Europa, lo que redujo la presencia de hambrunas en la mayor parte del continente (Fagan 2000). Por el contrario, la $\mathrm{PEH}$ fue descripta como una especie de breve "re-instalación" en las condiciones del último período glacial (caracterizada por el avance de numerosos glaciares en todo el mundo [Groove 2001]). Esto provocó que la mayor parte del continente europeo resultara inviable para la obtención de buenas cosechas durante varios años consecutivos y, en consecuencia, generó períodos de hambrunas y proliferación de pestes (Fagan 2000).

En la actualidad, los agentes forzantes involucrados en la consolidación de las condiciones imperantes durante la ACM se encuentran en discusión, incluyendo factores naturales como la radiación solar, el vulcanismo, la turbidez atmosférica (Díaz et al. 2011), la persistencia de una fase positiva en la anomalía recurrente conocida como Oscilación del Atlántico Norte (Trouet el al. 2009), y también las actividades humanas vinculadas al desarrollo de la producción agrícolo-ganadera en Europa (Goosse et al. 2006). En lo que hace a la PEH, existe cierto consenso en que la combinación de cambios en la Circulación Termohalina en el Atlántico Norte (que modera las temperaturas en Europa) y la reducción de la radiación solar (mínimo de Maunder [Eddy 1976]) jugaron un rol predominante en la consolidación de este período frío.

No obstante, los efectos de la ACM y la PEH, su duración, las fechas exactas de comienzo y fin de sus condiciones y su variabilidad interna parecen haber sido bastante diferentes en distintas partes del mundo (Díaz et al. 2011), y no necesariamente se ajustan a lo observado en Europa. Díaz et al. (2011) han sostenido que la categoría de ACM según Stine (1994, 1998) hoy en día resulta poco útil dada la heterogeneidad de condiciones y cronologías propuestas alrededor del mundo. En los Andes Centrales, una cantidad considerable de datos paleoambientales recuperados de registros lacustres $\mathrm{y}$, en particular, de los núcleos de hielo recuperados en el Glaciar Quelccaya (Thompson et al. 1986, 2006) y en el Nevado de Sajama (Bolivia) (Liu et al. 2005) han tenido una relevancia enorme para comenzar a definir las tendencias ambientales generales en la región durante las dos cronozonas.

No obstante, dada la escasez de información paleoambiental sobre los últimos 2000 años en los Andes en general, así como la variabilidad de resultados obtenidos en las distintas localidades estudiadas, resulta evidente la necesidad de incrementar la cantidad de registros paleoambientales que permitan estudiar el impacto de la ACM y de la PEH en diferentes sectores del noroeste argentino. Este trabajo presenta nueva información paleoambiental de alta resolución cronológica (decadal) para el sector noroeste de la Puna seca de Jujuy. La información referida corresponde al registro de diatomeas de un testigo del fondo de la Laguna Pululos, una laguna somera ubicada a 4500 m.s.n.m. Se presentan y discuten los resultados principales de este análisis, incluyendo la composición de los ensambles de especies de diatomeas y las tendencias paleoambientales que se observaron, relacionadas con las características y cronología de la ACM y la PEH.

\section{MATERIALES y MÉTODOS}

\section{Área de estudio}

La Puna es una enorme cuenca endorreica ubicada en los Andes Tropicales, por encima de 3000 m.s.n.m. Se la puede subdividir en cuencas menores interconectadas, que drenan en lagunas someras de agua dulce o salobre, en "vegas" o ciénagas y en salares y salinas. Éstos aumentan su frecuencia y extensión hacia el sector suroeste, siguiendo el gradiente de precipitaciones de la región. La característica predominante de la Puna es su aridez: la precipitación media es 200 mm/año, que varía desde $400 \mathrm{~mm} /$ año en el sector noreste de la Puna seca de Jujuy hasta $50 \mathrm{~mm}$ /año en el sector suroeste de la Puna salada catamarqueña. La región presenta una gran amplitud térmica diaria, con una temperatura media de $6{ }^{\circ} \mathrm{C}$.

En términos climáticos, Garreaud et al. (2003, 2009) indicaron que las condiciones medias en los Andes Tropicales están muy relacionadas con la circulación atmosférica de alto nivel (troposférica). Las precipitaciones de la región se encuentran vinculadas al balance entre los vientos del este (alisios) y los del oeste ("westerlies"), y a sus posiciones e intensidades respectivas. Mientras que la intensificación de los alisios produce el ingreso de humedad del Amazonas durante el verano (diciembre a marzo), en las tierras altas de los Andes, la intensificación o desplazamiento de los westerlies hacia el norte modera (e incluso 
bloquea temporalmente) el ingreso de humedad en la región, lo que en la zona produce eventos secos (Aceituno \& Montecinos 1993).

\section{Laguna Pululos}

Laguna Pululos $\left(22^{\circ} 32^{\prime} 43^{\prime \prime}\right.$ S; 66 $48^{\circ} 25^{\prime \prime}$ W) está ubicada a 4557 m.s.n.m. y es parte del Complejo lagunar Vilama (Figura 1), designado sitio Ramsar en el 2000. El Complejo Vilama tiene diversos cuerpos de agua someros, alimentados por vertientes y aguas de deshielo. Éstos incluyen cuerpos de agua dulce o salobre de tamaño pequeño a mediano (e.g., las lagunas Pululos, Caiti, Isla Grande y Arenal [Mirande \& Tracanna 2009]), y lagunas extensas muy salinas y alcalinas (e.g., Vilama y Palar). En las lagunas del complejo resultan comunes los depósitos eventuales de sedimentos de origen volcánico y las fluctuaciones marcadas en su profundidad y tenor salino (Caziani et al. 2001). Por el contrario, las características físicoquímicas de estas lagunas no presentarían impactos de origen antrópico ya que la población humana en el área actualmente es baja y, según sugiere la evidencia arqueológica, también parece haberlo sido en el pasado (Nielsen 2003).

La superficie de la Laguna Pululos es 990 ha. $\mathrm{Su}$ profundidad máxima es $1.2 \mathrm{~m}$, su agua es levemente salobre $(0.8 \%)$ y presenta abundante vegetación sumergida (Caziani \& Derlindati 1999). Las características físico-químicas de Pululos (Tabla 1) fueron relevadas por distintos equipos de investigación en diferentes momentos y con metodologías variadas, lo cual permite tener una idea del rango de variación de algunos parámetros ambientales.

Caziani \& Derlindati (1999) describieron las comunidades acuáticas de la Laguna Pululos sobre la base de muestras obtenidas en 1997; ellos enumeraron la presencia de Cyanobacteria, Chlorophyta, Bacillariophyceae y fitoflagelados (Euglenophyta y Cryptophyta) en muestras de fitoplancton y la presencia de cladóceros y copépodos en muestras de zooplancton. Por otra parte, se indicó la presencia de las macrófitas Myriophyllum sp., Zannichellia sp., Potamogeton sp. y Chara sp. Asimismo, Seeligman et al. (2008) y Maidana et al. $(2009,2011)$ describieron las

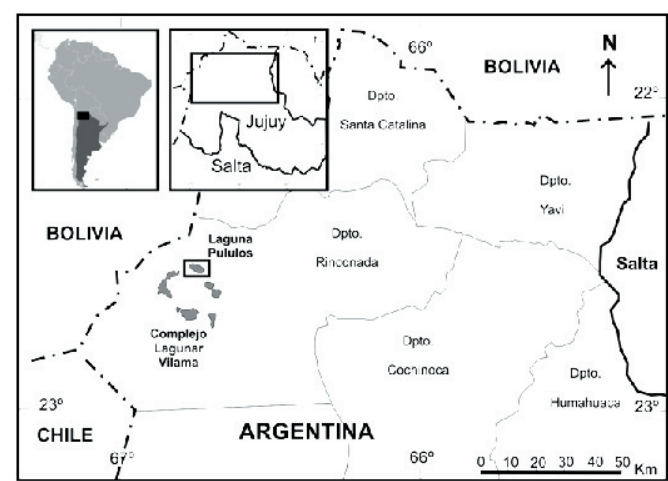

Figura 1. Mapa del área de estudio y sitio de muestreo. Figure 1. Map of the research area indicating the sampling site.

diatomeas a partir de muestras de la laguna colectadas en los años 2004 y 2005, y listaron especies como Cocconeis placentula, Halamphora veneta, Thalassiosira patagonica, Cratícula halophila, etc. A los fines de este estudio, consideramos que la flora de diatomeas contenida en el primer centímetro del testigo 1012 de la Laguna Pululos resulta una evidencia más representativa de la flora actual en el punto de muestreo.

\section{Cronología, colecta de muestras y técnicas de procesamiento}

En el año 1998, Martin Grosjean y colaboradores extrajeron un testigo de $120 \mathrm{~cm}$ de longitud (1012) de la margen de Laguna Pululos utilizando un muestrador tipo Livingstone. Para el análisis diatomológico el testigo se tomaron muestras cada $2 \mathrm{~cm}$. La secuencia temporal abarcada se determinó a partir de la datación radiocarbónica (AMS, accelerator mass spectrometry) de cinco muestras (Tabla 2). A partir de las fechas calibradas resultantes (SHCal04, southern hemisphere calibration curve 2004) (McCormac et al. 2004) se elaboró un modelo de edad/profundidad por interpolación lineal de los puntos medios de las fechas calibradas siguiendo a Bennett (1994), que resultó en la estimación de un intervalo de muestreo de $\sim 20$ años en el sector superior del testigo (desde $90 \mathrm{~cm}$ hasta la superficie) y de aproximadamente 52 años en el sector inferior, dada la compactación de los sedimentos.

Tabla 1. Datos fisico-químicos de la Laguna Pululos según diferentes estudios.

Table 1. Physicochemical data of Laguna Pululos according to different studies.

\begin{tabular}{|c|c|c|c|c|c|c|c|c|c|}
\hline & $\begin{array}{c}\text { Cond. } \\
(\mu \mathrm{S} / \mathrm{cm})\end{array}$ & $\begin{array}{l}\text { Alcalin. } \\
\text { (mg/L) }\end{array}$ & $\begin{array}{c}\text { Cloro } \\
(\mathrm{mg} / \mathrm{L})\end{array}$ & $\begin{array}{l}\text { Salin. } \\
(\mathrm{g} / \mathrm{L})\end{array}$ & $\begin{array}{c}\text { Sodio } \\
(\mathrm{mg} / \mathrm{L})\end{array}$ & $\begin{array}{l}\text { Sulfatos } \\
\text { (mg/L) }\end{array}$ & $\begin{array}{c}\text { Fósforo } \\
\text { total } \\
(\mathrm{mg} / \mathrm{L})\end{array}$ & $\mathrm{pH}$ & $\begin{array}{c}\text { Temp } \\
\left({ }^{\circ} \mathrm{C}\right)\end{array}$ \\
\hline Grosjean (1998; com. pers.) & 1694 & 237 & $\mathrm{~s} / \mathrm{d}$ & 0.8 & 237 & 285.6 & $\mathrm{~s} / \mathrm{d}$ & 8.9 & 18.6 \\
\hline Caziani \& Derlindati (1999) & 1380 & 290 & 166 & $\mathrm{~s} / \mathrm{d}$ & 178 & 250 & 1.3 & $\mathrm{~s} / \mathrm{d}$ & $\mathrm{s} / \mathrm{d}$ \\
\hline Seeligmann et al. (2008) & 1300 & $\mathrm{~s} / \mathrm{d}$ & $\mathrm{s} / \mathrm{d}$ & 0.9 & $\mathrm{~s} / \mathrm{d}$ & $\mathrm{s} / \mathrm{d}$ & $\mathrm{s} / \mathrm{d}$ & 9.2 & 16 \\
\hline Maidana et al. $(2009,2011)$ & 2990 & $\mathrm{~s} / \mathrm{d}$ & $\mathrm{s} / \mathrm{d}$ & 2.3 & $\mathrm{~s} / \mathrm{d}$ & $\mathrm{s} / \mathrm{d}$ & $\mathrm{s} / \mathrm{d}$ & 9.2 & 16 \\
\hline
\end{tabular}


Tabla 2. Dataciones radiocarbónicas obtenidas del testigo 1012 de Laguna Pululos.

Table 2. Radiocarbon dates obtained from core 1012 of Laguna Pululos.

\begin{tabular}{cccccc}
\hline $\begin{array}{c}\text { Profundidad } \\
(\mathrm{cm})\end{array}$ & $\begin{array}{c}\text { Código de } \\
\text { Laboratorio }\end{array}$ & Método & $\begin{array}{c}\text { Fecha } \\
\text { (años AP) }\end{array}$ & $\begin{array}{c}\text { Fecha cal. 10 } \\
\text { (años cal. DC) }\end{array}$ & $\begin{array}{c}\text { Fecha cal. 2ロ } \\
\text { (años cal. DC) }\end{array}$ \\
\hline 0 & Hv-22924 & ${ }^{14} \mathrm{C}$ & Moderna & & \\
48 & Hv-22716 & ${ }^{14} \mathrm{C}$ & $270 \pm 137$ & $1501-1810$ & $1464-1951$ \\
79 & Poz-11015 & AMS & $475 \pm 30$ & $1433-1463$ & $1420-1498$ \\
89 & Poz-14703 & AMS & $610 \pm 30$ & $1389-1412$ & $1382-1428$ \\
110 & Hv-22925 & ${ }^{14} \mathrm{C}$ & $1255 \pm 160$ & $680-977$ & $562-1159$ \\
\hline
\end{tabular}

Para el tratamiento del material se siguieron los procedimientos estándar sugeridos por Battarbee (1986): oxidación de la materia orgánica con $\mathrm{H}_{2} \mathrm{O}_{2}$ al 30\% y calor, eliminación de carbonatos con $\mathrm{HCl}$ $10 \%$ y neutralización de la suspensión resultante mediante lavados sucesivos con agua destilada. El material tratado fue montado en portaobjetos de manera permanente con Naphrax®. Se identificaron y contaron alrededor de 400 valvas de diatomeas por muestra, utilizando un microscopio binocular a 1000x. Para confirmar la asignación taxonómica de los individuos más pequeños se utilizó un microscopio electrónico de barrido (Phillips XL30 $\mathrm{TMP}$ ), previo metalizado de las muestras con oro-paladio en el Museo Argentino de Ciencias Naturales Bernardino Rivadavia (Buenos Aires). Las determinaciones taxonómicas se realizaron siguiendo trabajos monográficos de Krammer \& Lange-Bertalot (1986, 1988, 1991a, 1991b), Round et al. (1990), Rumrich et al. (2000) y LangeBertalot (2001), entre otros. Las zonas florísticas se determinaron mediante el programa CONISS (Grimm 1987) que utiliza un análisis de cluster constreñido estratigráficamente.

La información sobre las diferentes formas de vida se obtuvo de los trabajos monográficos de Luchini \& Verona (1972), Lowe (1974), DeWolf (1982) y Van Dam et al. (1994), y sobre taxones específicos siguiendo a Vos \& DeWolf (1993), Willie et al. (2007) y Levkov (2009), entre otros. Con estas afinidades se construyó un índice de humedad (IH) basado en el cociente entre las abundancias relativas de las especies planctónicas, ticoplanctónicas y epifitas, y las de las aerófilas y bentónicas. Este índice es comparable a otros utilizados en trabajos previos en ambientes comparables (Morales 2011; Tchilingurian et al. 2013, 2014). Los valores de IH obtenidos a partir de abundancias presentes en las muestras se transformaron mediante logaritmos y se graficaron para comparar con la media de la serie a fin de definir los períodos más húmedos y más secos de los últimos 1400 años.

\section{Resultados}

Se identificaron 91 taxones a nivel de especie y variedad; los más frecuentes fueron Cocconeis placentula Ehrenberg, Thalassiosira patagonica Maidana, Cyclotella meneghiniana Kützing y Halamphora veneta (Kützing) Levkov. En la Figura 2 se presentan las abundancias relativas de los 15 taxones que superan el 5\% en al menos una de las muestras, a lo largo del registro del testigo 1012, agrupadas por forma de vida. A partir de la composición de la flora se identificaron tres zonas: A, B y C. Las zonas A y C fueron, a su vez, divididas en dos subzonas. La zona C2 (entre 600 y 700 DC) presentó frecuencias altas de T. patagonica, seguida por C. placentula y bajas frecuencias de otros taxones (en particular de Navicula spp.), registradas regularmente en el resto del testigo. En la zona C1 (aproximadamente 700 y 1450 años DC) predominaron C. placentula, T. patagonica y $H$. veneta; además, presentó frecuencias moderadas, aunque estables, de los géneros Hippodonta, Navicula y Nitzschia. La zona B (entre $\sim 1450$ y 1660 DC) se caracterizó por la transición desde la dominancia casi absoluta de la especia planctónica T. patagonica ( 1550 DC) hacia la dominancia de C. placentula, acompañada de otros taxones litorales como Gomphonema punae Lange-Bertalot \& Rumrich y Gomphonema clavatum Ehrenberg. La zona A2 (entre 1660 y 1880 DC) se caracterizó por la dominancia de C. placentula y la co-dominancia de $C$. meneghiniana y $H$. veneta, acompañadas por T. patagónica, C. halophila y de los géneros Navicula, Nitzschia y Gomphonema, todos con baja frecuencia en los momentos más tempranos de la zona. Por su parte, en la zona A1 (entre 1880 y 2000 DC) co-dominaron C. meneghiniana y C. placentula, acompañadas por bajas frecuencias de T. patagonica, Craticula halophila (Grunow) D. G. Mann y H. veneta, y algunos escasos representantes de los géneros Navicula, Nitzschia y Gomphonema.

Los valores obtenidos para el IH (entre 0.036 y 77.724) y la agrupación de los taxones en función de sus formas de vida permitieron separar las muestras en tres momentos ambientales (Figura 3):

1) desde $\sim 600$ DC hasta $\sim 800 \mathrm{DC}$, con un IH mayor a la media de la serie (7.155) y la predominancia de diatomeas planctónicas 


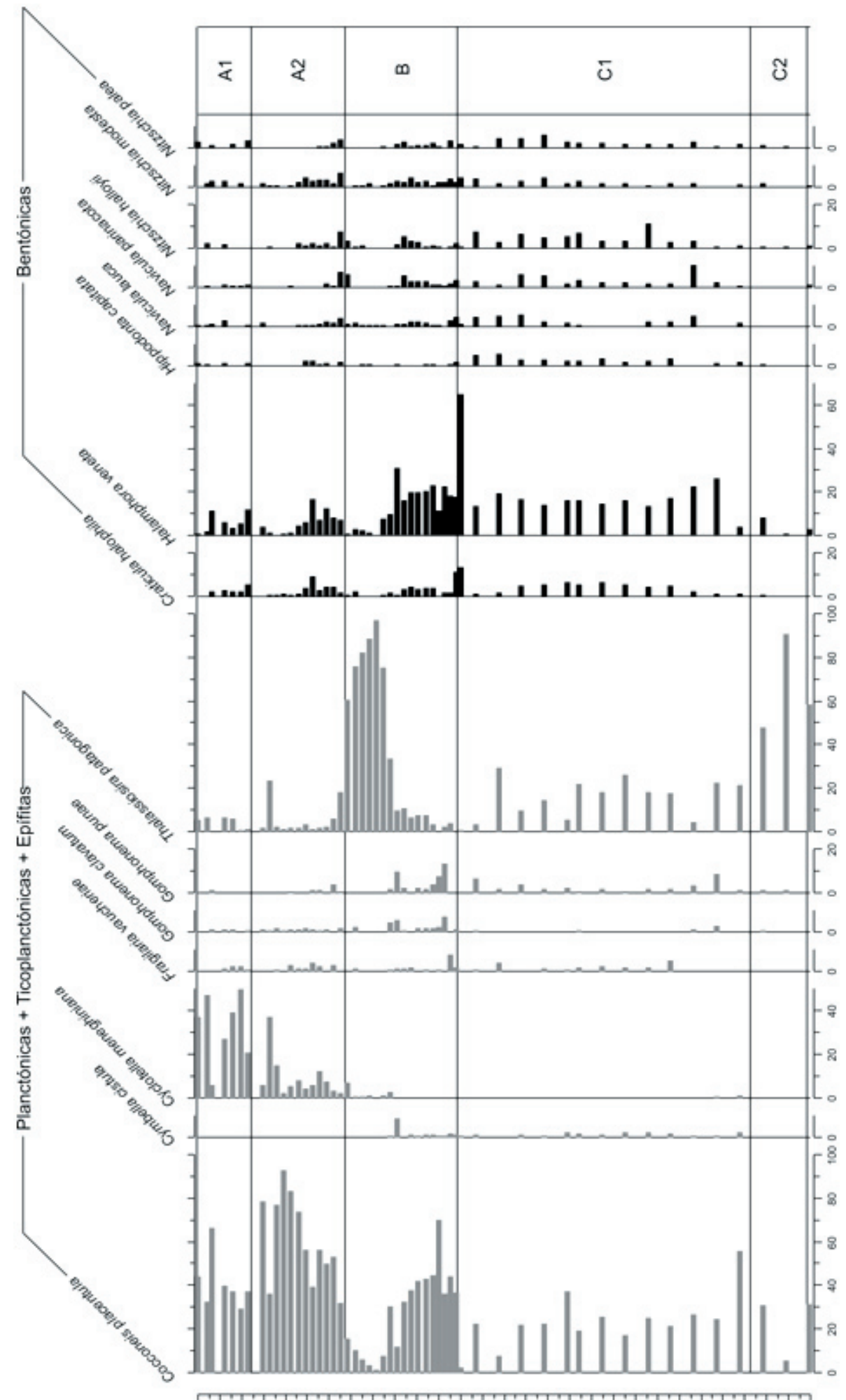

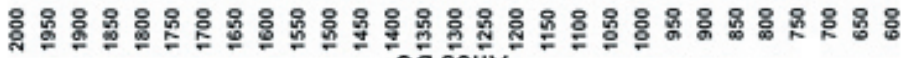

Figura 2. Frecuencia, forma de vida y zonación de las especies de diatomeas (>5\%) en el testigo 1012 de Laguna Pululos.

Figure 2. Frequency, life form and zonations of diatom species ( $>5 \%)$ in core 1012 of Laguna Pululos. 
2) desde $\sim 800$ hasta $\sim 1500$ DC, con valores de IH por debajo de la media, con el valor mínimo de la serie (0.036) alrededor del año 1400 DC, y con la dominancia de diatomeas bentónicas, $\mathrm{y}$

3) desde 1500 hasta 1998 DC, con mayoría de valores de IH por encima de la media de la serie y algunos por debajo (en un rango de 0.708 a 77.724), con predominancia las diatomeas epífitas y planctónicas. Cabe resaltar un evento puntual de unos 100 años de duración (entre 1550 y 1650 DC) en el que se observa un aumento del nivel de la laguna, comparable a los niveles alcanzados entre los años 600 y 800 DC.

\section{DisCUSIÓN}

A través del análisis de la composición florística de las muestras investigadas hemos identificado tres conjuntos de especies dominantes que sugieren condiciones ambientales distintas. El primer conjunto fue dominado por Thalassiosira patagonica, una pequeña diatomea planctónica hallada por primera vez tanto en material actual como en sedimentos cuaternarios de lagos de la Patagonia austral argentina (Maidana \& Corbella 1997; Maidana 1999). También fue identificada en sedimentos recientes de una laguna pampeana (Miretzky et al. 2002) y en lagunas de altura del noroeste argentino, de Bolivia (Sylvestre, comunicación personal) y de Perú (Tapia, comunicación personal). Se la considera como indicadora de ambientes lacustres con conductividades relativamente altas (Wille et al. 2007). Así, su amplia dominancia en las muestras de Laguna Pululos indica niveles altos en la laguna con eventos de concentración de sales debido a períodos de mayor aridez. El segundo conjunto lo constituyeron muestras co-dominadas por Cocconeis placentula, Halamphora veneta y Thalassiosira patagonica (en frecuencias variables). C. placentula es una especie cosmopolita, típicamente epífita o ticoplanctónica. Por su parte, $H$. veneta es bentónica, con una distribución geográfica amplia en cuerpos de agua de dulces a levemente salobres, hallada frecuentemente en ambientes eutróficos (Levkov 2009). Esta composición sugiere un cuerpo de agua somero, algo más profundo y vegetado, a mayor frecuencia de T. patagónica y $C$. placentula, respectivamente, o menos profundo y eutrófico a mayor frecuencia de $H$. veneta. El último conjunto estuvo constituido por las muestras co-dominadas por Cocconeis placentula y Cyclotella meneghiniana, esta última de tipo planctónica o ticoplanctónica, cosmopolita, de aguas alcalinas dulces o levemente salobres (Luchini \& Verona 1972; Lowe 1974), indicadoras de un ambiente alcalino con una zona litoral amplia. Cabe mencionar que las especies que componen estos ensambles fueron halladas también en

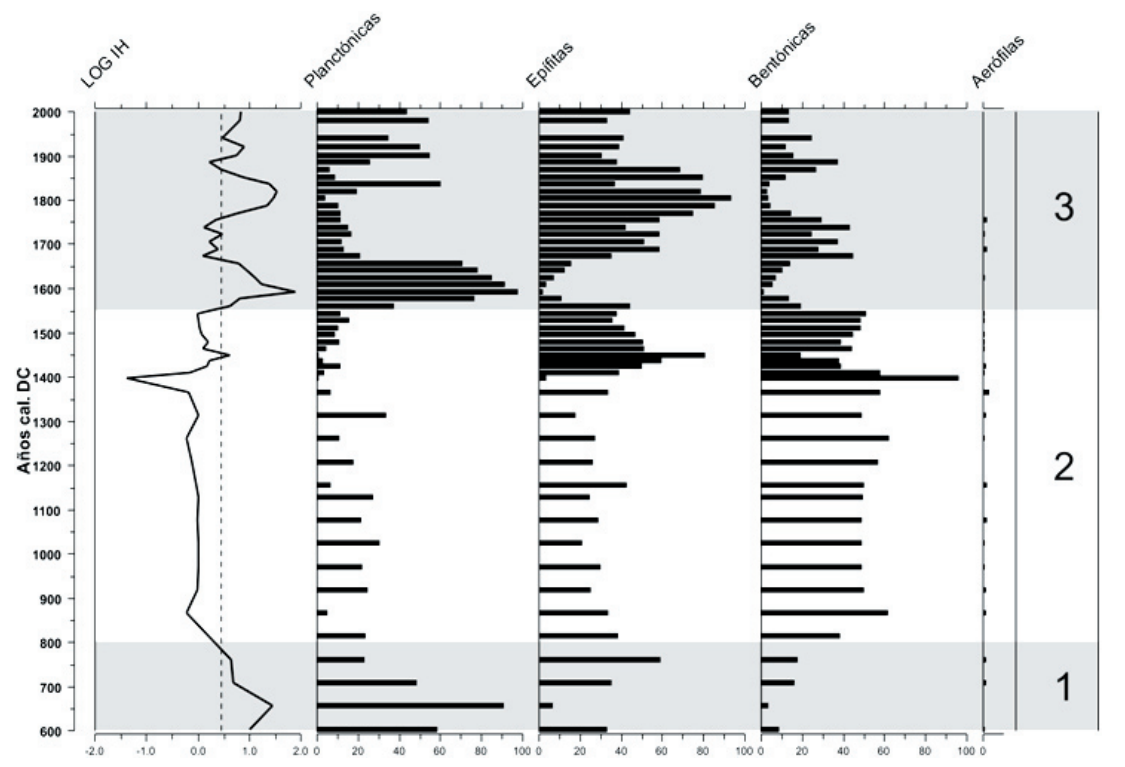

Figura 3. Índice de Humedad (IH, escala logarítmica) y frecuencia de las distintas formas de vida de las diatomeas del testigo 1012 de Laguna Pululos, que permiten distinguir los tres momentos ambientales del registro.

Figure 3. Moisture Index (MI, log scale) and frequency of the different diatoms life forms of core 1012 of Laguna Pululos, allowing to distinguish three environmental moments in the record. 
muestras actuales (Seeligmann et al. 2008; Maidana et al. 2009), excepto Cyclotella meneghiniana. Asimismo, todas estas especies se encuentran conjuntamente en la primera muestra del testigo 1012, que representaría las últimas dos décadas del registro, aunque T. patagónica y, particularmente, $H$. veneta presentaron baja frecuencia.

\section{Sintesis paleoambiental de los ensambles de diatomeas de Laguna Pululos}

En conjunto, la información sobre la composición porcentual de los ensambles de diatomeas precedentes y el índice de humedad calculado para el testigo 1012 permitió reconocer la existencia de períodos con diferentes condiciones ambientales en la Laguna Pululos (Figura 3). El primer período comienza en la base de la secuencia ( $~ 600$ DC) y finaliza 200 años después ( 800 DC). Las muestras de este lapso se encuentran dominadas por Thalassiosira patagonica, acompañada por Cocconeis placentula, lo que sugiere condiciones algo salinas con amplia zona litoral, y probablemente la máxima profundidad registrada en este sistema. En el segundo período, las condiciones aparentemente sufren un cambio drástico en menos de 100 años, con el establecimiento del período más árido del registro entre $\sim 900$ y 1500 DC. En ese período, la Laguna Pululos presentaría poca profundidad y condiciones eutróficas. Su flora de diatomeas se caracterizó por una frecuencia relativamente baja de $T$. patagonica y una dominancia de formas bentónicas como $H$. veneta y Navicula spp. y Craticula spp. Durante este extenso período seco se observó una fase árida de 100 años de duración entre 1366 DC y 1463 DC, según se deduce por la caída en la frecuencia de diatomeas planctónicas y el incremento de especies bentónicas. Esto generó los valores más bajos en el índice de humedad. Después de este pico de aridez las condiciones de humedad habrían comenzado a cambiar, permitiendo el desarrollo de formas epífitas como C. placentula y distintas especies de Gomphonema.

Nuevamente, en un breve lapso de dos o tres décadas, las características del cuerpo de agua habrían cambiado de forma sustancial. Hacia 1544 DC se observó un fuerte cambio en el balance hídrico del sistema, en coincidencia con el final de la zona B (Figura $2)$, caracterizado por la instalación de un ambiente más profundo y menos salino, con eventos recurrentes de desecación, que aparentemente se mantuvo hasta el año 1690 DC. Este período presentaría condiciones comparables a las registradas entre los años 600 y 800 DC. A partir de esta fecha, y aproximadamente hasta el año 1869 DC, la flora de diatomeas indica condiciones de aguas más dulces y niveles más estables. Esto explicaría el reemplazo de $T$. patagonica por C. meneghiniana y la desaparición de halófilas como Chaetoceros muelleri, ya que una menor frecuencia de eventos de aridez (tolerados por T. patagonica y en los que prolifera C. muelleri) favorecerían el desarrollo de C. meneghinia, de macrófitas y de vegetación litoral asociada a especies epífitas o ticoplanctónicas como $C$. placentula.

Desde 1869 DC hasta el presente, la laguna habría sido más dulce y con una amplia zona litoral, como lo sugiere la co-dominancia de C. meneghiniana y de C. placentula. De todas formas, la permanencia de T. patagonica en bajas frecuencias sugiere que sucedieron de manera recurrente eventos anuales o estacionales, o breves lapsos plurianuales, con condiciones algo salobres.

\section{Implicancias de los resultados paleoambientales en lo referido a la ACM y la PEH}

Si se comparan las tendencias ambientales observadas para los últimos 1400 años en Laguna Pululos con la información disponible para los Andes Tropicales (Thompson et al. 1985, 1986, 2006; Abbott et al. 2003; Jomelly et al. 2009; Liu et al. 2005; Morales et al. 2012) es posible observar dos rasgos comunes en muchos de los registros estudiados. Por un lado, la presencia de condiciones de marcada aridez que finalizan entre 1300 y 1500 DC y por otro, la instalación de condiciones más húmedas a partir de esta fecha y hasta fines del siglo XIX.

Con respecto a la instalación de condiciones de aridez asimilables a la ACM en el área andina, la información es variada entre los registros. Mientras que el nivel de agua del Titicaca parecería decrecer hacia el año 1000 DC, las estimaciones de las precipitaciones en la laguna Pumacocha, en Perú (Abbott et al. 2003), ubican un lapso de aridez marcada, asimilado a la ACM el período entre el 900 y el 1100 DC. Por su parte, los indicadores analizados en Quelccaya ubican el comienzo de estas condiciones hacia el 1100 DC (Thompson et al. 1985, 1986, 2006). Los ensambles de diatomeas de Laguna Pululos indican un inicio algo más temprano de estas condiciones de 
sequía, en torno al 900 DC, coincidiendo con las fechas de laguna Pumacocha.

El fin de este período de aridez parecería perdurar algo más en el registro de Laguna Pululos, en comparación con los otros registros disponibles (Abbott et al. 2003; Thompson et al. 1985, 1986, 2006), extendiéndose hasta 1550 DC. Además, los registros indican un evento extremo de sequía y una máxima contracción de la laguna entre 1350 y 1450 DC. Estas condiciones de aridez se extenderían algo más allá que en el Lago Titicaca, donde se observó una recuperación del nivel a partir de 1350 DC (Abbott et al. 2003). El evento de sequedad extrema de Laguna Pululos resulta algo posterior al lapso de mayor temperatura y aridez detectado en el glaciar Quelccaya entre 1100 y 1300 DC (Thompson et al. 1985, 1986, 2006). Además, coincide con la reducción en las precipitaciones entre 1300 y 1450 DC indicada por el análisis de anillos de crecimiento de Polilepis tarapacana, recuperados próximos a Laguna Pululos (Morales et al. 2012).

Las condiciones de mayor humedad registradas luego del 1500 DC y hasta fines del siglo XIX ( 1880) en la Laguna Pululos coinciden con buena parte de los registros estudiados en los Andes Tropicales (Thompson et al. 1985, 1986, 2006) y con algunos del hemisferio norte (Knoll et al. 1979; Mann 2002). Resulta notable la coincidencia de los eventos de avance glacial de los últimos 1000 años, entre 1630 y 1680 DC y entre 1730 y 1890 DC (Jomelly et al. 2009) con los momentos más húmedos de Laguna Pululos. Cabe destacar que entre los años 1550 y 1650 DC se produjo un evento de humedad en Laguna Pululos en coincidencia con el evento deextensión máxima de avance glaciar (1630-1680 DC) (Jomelly et al. 2009). Vale mencionar, asimismo, la falta de una señal clara del primer pulso glaciar en la laguna, registrado hacia 1250 DC (Jomelly et al. 2009). Por su parte, la evidencia polínica del Nevado de Sajama (Liu et al. 2005) resulta coincidente con la información precedente y con los valores isotópicos de Quelccaya, que indican condiciones frías durante el período 1500-1880 DC (Thompson et al. 1986). Sin embargo, en ninguno de los registros mencionados se observa la alternancia entre un fase húmeda (entre 1500-1700 DC) y una comparativamente más seca (entre 1700 y 1880 DC) observada en Sajama por Liu (2005).

El registro de Pululos también muestra algunas diferencias importantes con otros registros del área en lo que hace a las condiciones dominantes durante el siglo XX. En el registro de Laguna Pululos se infieren condiciones relativamente húmedas, lo cual sugiere un cuerpo de agua dulce somero, estable y con una amplia zona litoral como en la actualidad. Sin embargo, se han propuesto para la región en ese lapso precipitaciones muy escasas, comparables con las condiciones más áridas de la ACM (Morales et al. 2012). En virtud de esta discrepancia, consideramos que es factible sostener a modo de hipótesis que el cambio en las especies de diatomeas planctónicas dominantes, que se hace manifiesto hacia fines del siglo XIX, se debe a la mayor flexibilidad ecológica de C. meneghiniana. Esta especie cosmopolita presentaría una ventaja selectiva en un ambiente más inestable y sujeto a desecaciones más frecuentes que las ocurridas durante la mayor parte de la PEH.

\section{Conclusiones}

Las cinco zonas florísticas identificadas en el testigo 1012 pueden ser resumidas en tres grandes momentos ambientales (Figura 3) que habrían sucedido con transiciones relativamente abruptas (lapsos menores a 100 años): a) un momento más húmedo entre 600 y 800 DC, que puede haber constituido el lapso de mayor profundidad del cuerpo de agua; b) un período mucho más árido y relativamente estable entre 800 y 1550 DC, cuando Laguna Pululos se habría comportado como una laguna muy somera y más salobre. Durante este período habría tenido lugar un evento árido entre 1350 y 1450 DC; y c) desde 1550 DC hasta el presente, cuando se habrían instalado condiciones más húmedas hasta el final de la secuencia. Estás condiciones implicaron un nivel mayor de la laguna y se habrían iniciado con un pulso húmedo entre 1550 y 1650 DC, comparable con el detectado entre el 600 y el 800 DC, para tornarse luego más variables, en particular en el período 1650-1700 DC.

La conjunción de los resultados obtenidos en L. Pululos con los disponibles para el resto de los Andes Tropicales indica que los patrones descriptos para la ACM en el hemisferio norte deben ser ajustados tanto en su duración como en su cronología para incluir a los Andes Tropicales. Al respecto, la presencia de condiciones de marcada aridez en la mayor parte de las localidades estudiadas en los Andes Tropicales entre 800 y 1500 DC permitiría extender en más de 200 años la 
crono-zona de la ACM en comparación con el hemisferio norte. En el caso de Laguna Pululos en particular, los resultados obtenidos indican un momento con características asimilables a la ACM con esta cronología, aunque con un pico de máxima aridez acotado a unos 100 años entre 1360 y 1460 DC.

Por su parte, la PEH parece presentar una cronología más uniforme y características menos heterogéneas que la ACM en la mayor parte de los registros estudiados hasta el momento en los Andes Tropicales, entre los cuales se encuentra el de Laguna Pululos.

Agradecimientos:Agradecemos a Martin Grosjean la oportunidad de analizar los sedimentos recuperados por él en Laguna Pululos y por facilitarnos amablemente las dataciones y otros datos del testigo. A los dos evaluadores anónimos del manuscrito por sus útiles comentarios y sugerencias. A la ANCPCyT por el subsidio FONDCyT PICT 20112352 que financió parcialmente los análisis del testigo

\section{BILBIOGRAFÍA}

Аввott, M; B Wolfe; A Wolfe; G Seltzer; R Aravena; et AL. 2003. Holocene Palehydrology and glacial history of the central Andes using multiproxy lake sediments studies. Palaeogeography, palaeoclimatology, palaeoecology, 194:123-138.

Aceituno, P \& A Montecinos. 1993. Análisis de la estabilidad de la relación entre la Oscilación del Sur y la precipitación en América del Sur. Bulletin de l'institut Francais d'Etudes Andines, 22(1):53-64.

Battararbee, RW. 1986. Diatom analysis. Pp. 527-570 en: Berlund, BE (ed.). Handbook of Holocene Paleoecology and Palaeohydrology. J Wiley \& Sons Ltd. New York.

BENNETT, KD. 1994. Confidence intervals for age estimates and deposition times in late-Quaternary sediment sequences. The Holocene, 4(4):337-348.

CaZiani, SM \& EJ Derlindati. 1999. Humedales altoandinos del Noroeste de Argentina: su contribución a la biodiversidad regional. Capítulo 1. Pp. 1-13 en: Tópicos sobre humedales subtropicales y templados de Sudamérica. Oficina Regional de Ciencia y Tecnología de la UNESCO para América Latina y el Caribe-ORCYT-Montevideo. Uruguay.

Caziani, S; E Derlindati; A Tálamo, Al Sureda; C Trucco; ET AL. 2001. Waterbird Richness in Altiplano Wetlands of Nothwestern Argentina. Waterbirds, 24(1):103-117.

DEWolf, H. 1982. Method of coding of ecological data from diatoms for computer utilization. Mededel. Rijks Geol. Dienst., 36(2):95-110.

Díaz, HF; R. TRigo; MK Hughes; ME ManN; E Xoplaki; ET AL. 2011. Spatial and temporal characteristics of climate in medieval times revisited. Bulletin of the American Meteorological Society, 92(11):1487-1500.

FAGAN, BM. 2000. The Little Ice Age: how climate made history, 1300-1850. Basic Books.

Garreaud, RD; M Vuille \& AC Clement. 2003. The climate of the Altiplano: observed current conditions and mechanisms of past changes. Palaeogeography, Palaeoclimatology, Palaeoecology, 194(1-3):5-22.
Garreaud, RD; MVuIlle, RCOMPAGNucci \& J Marengo. 2009. Present-day South American climate. Palaeogeography, Palaeoclimatology, Palaeoecology, 281(3-4):180-195.

Goose, H; O Arzel; J Luterbacher; ME ManN; H Renssen; ET AL. 2006. The origin of the European „Medieval Warm Period". Climate of the Past, 2:99-113.

GRIMM, EC. 1987. CONISS: A Fortran 77 program for stratigraphically constrained cluster analysis by the method of incremental sum of squares. Computers $\mathcal{E}$ Geosciences, 13(1):13-35.

Grove, J. 2001. The onset of Little Ice Age. Pp. 153-185 en: Jones, PD; AEJ Ogilvie; TD Davies \& KR Briffa (eds.). History and Climate: Memories of the Future? Kluwer Academic / Plenum Publishers, New York.

KNOLL, AH; KJ NiKLAS \& BH TiffNey. 1979. The" Little Ice Age": Northern hemisphere average observations and model calculations. Science, 206:1402.

KRAMMER, K \& H LANGE-BERTALOT. 1986. Bacillariophyceae. 1. Teil: Naviculaceae. En: Ettl, H; G Gärtner; J Gerloff; H Heynig \& D Mollenhauer (eds). Süsswasser ora von Mitteleuropa. 2(1):1-876. Gustav Fischer Verlag: Stuttgart, Jena.

Krammer, K \& H Lange-Bertalot. 1988. Bacillariophyceae 2. Teil: Bacillariaceae, Epithemiaceae, Surirellaceae. En: Ettl, H; G Gärtner; J Gerloff; H Heynig \& D Mollenhauer (eds). Süßwasserflora von Mitteleuropa, 2(2):1-596. Gustav Fischer Verlag: Stuttgart, Jena.

KRAMMER, K \& H LANGE-Bertalot. 1991a. Bacillariophyceae 3. Teil: Centrales, Fragilariaceae, Eunotiaceae. En: Ettl, H; G Gärtner; J Gerloff; H Heynig \& D Mollenhauer (eds). Süßwasserflora von Mitteleuropa, 2(3):1-576. Gustav Fischer Verlag: Stuttgart, Jena.

Krammer, K\& H LANGe-Bertalot. 1991b. Bacillariophyceae. 4. Teil: Achnanthaceae Kritische Ergänzungen zu Navicula (Lineolatae) und Gomphonema. En: Ettl, H; G Gärtner; J Gerloff; H Heynig \& D Mollenhauer (eds). Süßwasserflora von Mitteleuropa, 4:1-437. Gustav Fischer Verlag: Stuttgart, Jena.

LANGE-BERTALOt, H. 2001. Navicula sensu stricto. 10 Genera Separated from Navicula sensu lato. Frustulia.. Diatoms of Europe, 2:1-526. ARG Gantner Verlag.

LAMB, HH. 1965. The early Medieval warm epoch and its sequel. Palaeogeography, Palaeoclimatology, Palaeoecology, 1:13-37.

LiU, KB; CA ReEse \& LG ThOMPSON. 2005. Ice-core pollen record of climatic changes in the central Andes during the last 400 yr. Quaternary Research, 64(2):272-278.

LowE, RL. 1974. Environmental requirements and pollution tolerance of freshwater diatoms. Environmental Research Center, U. S. Environmental Protection Agency. Cincinnati, Ohio.

MaIdAna, NI. 1999. Thalassiosira patagonica sp. nov. (Thalassiosiraceae, Bacillario-phycaea), a new lacustrine centric diatom from Santa Cruz, Argentina. Diatom Research, 14(2):323-329.

Maidana, NI \& H CoRbella. 1997. Análisis preliminar de las asociaciones de diatomeas cuaternarias en un paleolago volcánico, Santa Cruz austral, Argentina. Pp. 336-340 en: Actas del VI Congresso Brasileiro de Abequa, Curitiba, Brasil.

Maidana, Ni; C Seeligman \& MR Morales. 2009. Bacillariophyceae del Complejo Lagunar Vilama (Jujuy, Argentina). Bol. Soc. Argent. Bot., 44(3-4):257-271.

Maidana, NI; C SEeligman \& MR Morales. 2011. El género Navicula sensu stricto (Bacillariophyceae) en humedales 
de altura de Jujuy, Argentina. Bol. Soc. Argent. Bot., 46(1-2):13-29.

ManN, ME. 2002. Little ice age. Encyclopedia of global environmental change, 1:504-509.

McCormac, FG; AG HogG; PG Blackwell; CE Buck; TF Higham; ET AL. 2004. SHCal04 Southern Hemisphere calibration, 0-11.0 cal kyr BP. Radiocarbon, 46(3):10871092.

Mirande, V \& BC Tracanna. 2009. Estructura y controles abióticos del fitoplancton en humedales de altura. Ecología Austral, 19(2):119-128.

Miretzky, P; NI Maidana \& A Fernández Cirelli. 2002. Stability of diatom composition in a variable lake environment: Lake Chascomús, Argentina. Limnology, 3(2):77-85.

Morales, MS; DA Christie; R Villalba; J Argollo; J Pacajes; ET AL. 2012. Precipitation changes in the South American Altiplano since 1300 AD reconstructed by tree-rings. Climate of the Past, 8(2):653-666.

Round, FE; RM CRAWFORD \& DG MANN. 1990. The Diatoms. Biology and Morphology of the genera. Cambridge University Press. Cambridge

Rumrich, U; H Lange-Bertalot \& M Rumrich. 2000. Diatomeen der Andenvon Venezuel a bis Patagonien/ Tierra del Fuego. En: Lange-Bertalot, $\mathrm{H}$ (ed.) Iconographia Diatolmologica 9. ARG Gantner Verlag KG. Germany. Pp. 672.

Seeligman, CT; Ni Maidana \& MR Morales. 2008. Diatomeas (Bacillariophyceae) de Humedales de Altura de La Provincia de Jujuy-Argentina. Bol. Soc. Argent. Bot., 43(1-2):1-17.

Stine, S. 1994. Extreme and persistent drought in California and Patagonia during mediaeval time. Nature, 369:546-549.

STINE, S. 1998. Medieval climatic anomaly in the Americas. Pp. 43-67 en: Water, Environment and Society in Times of
Climatic Change. Springer. Netherlands.

Thompson, LG; E Mosley-Thompson; JF BolzAn, J \& BR Koci. 1985. A 1500-year record of tropical precipitation in ice cores from the Quelccaya ice cap, Peru. Science, 229(4717):971-973.

THOMPSOn, LG; E Mosley-Thompson; W DANSGAard \& PM Grootes. 1986. The Little Ice Age as recorded in the stratigraphy of the tropical Quelccaya ice cap. Science, 234(4774):361-364.

Thompson, LG; E Mosley-Thompson; H BReCher; M DAVIS; B LEÓN; ET AL. 2006. Abrupt tropical climate change: Past and present. Proceedings of the National Academy of Sciences, 103(28):10536-10543.

Trouet, V; J Esper; NE Graham; A Baker; JD Scourse; et AL. 2009. Persistent positive North Atlantic Oscillation mode dominated the medieval climate anomaly. Science, 324(5923):78-80

Valero-Garcés, BL; A Delgado-Huertas; A Navas; L EDWARDS; A SCHWALB; ET AL. 2003. Patterns of regional hydrological variability in central-southern Altiplano (18-26 S) lakes during the last 500 years. Palaeogeography, Palaeoclimatology, Palaeoecology, 194(1):319-338.

Van Dam, H; A Mertens \& J Sinkeldam. 1994. A coded checklist and ecological indicator values of freshwater diatoms from the Netherlands. Netherland Journal of Aquatic Ecology, 28(1):117-133.

Vos, PC \& H DeWolf. 1993. Diatoms as a tool for reconstructing sedimentary environments in coastal wetlands; methodological aspects. Pp. 285-296 en: Twelfth International Diatom Symposium. Springer, Netherlands.

Wille, M; NI Maidana; F Schäbitz; M Fey; T Haberzettl; ET AL. 2007. Vegetation and climate dynamics in southern South America: the microfossil record of Laguna Potrok Aike, Santa Cruz, Argentina. Review of Palaeobotany and Palynology, 146:234-246. 